\title{
TURISMO RELIGIOSO Y ACCESIBILIDAD UNIVERSAL: COMPATIBILIDADES E INCOMPATIBILIDADES ${ }^{1}$
}

\section{RELIGIOUS TOURISM AND UNIVERSAL ACCESSIBILITY: COMPATIBILITIES AND INCOMPATIBILITIES}

\section{TURISMO RELIGIOSO E ACESSIBILIDAD UNIVERSAL: COMPATIBILIDADES E INCOMPATIBILIDADES}

\author{
${ }^{2}$ Maria Abril-Sellarés
}

\section{RESUMEN}

Los edificios monumentales que se encuentran en los centros históricos y, que se corresponden a destinos maduros, a través del turismo cultural se han convertido en "objetos visitables" y por tanto en recurso turístico, que según su valor, histórico, estético o ideológico atraen a más o menos flujos turísticos. Las Catedrales pueden, sin lugar a dudas, seducir por esos tres valores a una idiosincrasia de visitantes, pero ¿son siempre accesibles para el cliente-turista? Este artículo tiene como objetivo analizar la gestión que llevan a cabo las Catedrales de Barcelona, Girona y Palma de Mallorca, en cuanto a temas de turismo accesible se refiere. Los objetivos que se quieren alcanzar son tres: el primero dilucidar cómo gestionan las Catedrales los problemas de motricidad del público en general; el segundo tener conocimiento sobre qué líneas de accesibilidad se ha hecho más hincapié: motriz, sordera o ceguera o si se han gestionado todas por un igual; y finalmente, el tercer objetivo es ver en qué grado se ha hecho la adaptación, si de un modo total o parcial. Para aseverar o refutar dichos objetivos la metodología utilizada ha sido doble, por una parte un análisis y revisión documental de la literatura científica y por otra un análisis cuantitativo - cualitativo a través de un cuestionario llevado a cabo en los recursos patrimoniales objeto de esta investigación.

\footnotetext{
${ }^{1}$ Este documento forma parte de una investigación llevada a cabo por el grupo de investigadores procedentes de las Universidades de Barcelona, Palma de Mallorca y la Coruña

2 Doctora por la Universidad de Barcelona en Didáctica de las Ciencias Sociales y del Patrimonio. E-mail: marabse@gmail.com
} 
Palabras Clave: Catedral, Destino, Recurso, Accesibilidad Universal, Discapacidad.

\section{ABSTRACT}

The monumental buildings found in the historic centers and, corresponding to mature destinations, through cultural tourism have become "objects to visit" and therefore tourist resort, which according to its value, historical, aesthetic or ideological appeal more or less tourist flows. Cathedrals can, without doubt, attracted by the three values to an idiosyncrasy of visitors, but are always accessible to the client-tourist? This article aims to analyze the management holding the Cathedrals of Barcelona, Girona and Palma de Mallorca, in terms of issues of accessible tourism is concerned. The objectives to be achieved are three: the first one figure out how to manage the Cathedrals motor skill problems to the public in general; the second one to have knowledge about which availability line has been more emphasis: driving, deafness or blindness or has handled all an equal; and finally, the third one is to know what extent has become, if a full or partial, adaptation. To assert or refute these objectives the methodology used has been twofold, on the one hand a documentary analysis and review of the scientific literature and on the other a quantitative - qualitative analysis through a questionnaire carried out in the patrimonial resources object of this investigation

Key Words: Cathedral, Destination, Resources, Universal Accessibility, Disability

\section{RESUMO}

Os edifícios monumentais encontrados nos centros históricos e, o que corresponde a amadurecer destinos, através do turismo cultural tornaram-se "objetos visitáveis" e, portanto, um recurso turístico, que de acordo com o seu valor, o apelo histórico, estético ou ideológico atraem mais ou menos fluxos turísticos. Catedrais são, sem dúvida, podem seduzir por esses três valores para uma idiossincrasia de visitantes, mas ¿estão sempre acessíveis para o clienteturista? Este artigo tem por objetivo analisar a gestão segurando as catedrais de Barcelona, Girona e Palma de Mallorca, em termos de questões de turismo acessível se refere. Os 
Turismo Religioso Y Accesibilidad Universal: Compatibilidades e Incompatibilidades

objectivos a atingir são três: o primeiro descobrir como gerenciam as catedrais os problemas de mobilidade do público em geral; o segundo a ter conhecimento sobre qual a disponibilidade de linha tem sido mais ênfase: condução, surdez ou cegueira ou têm sido geridos todos para o mesmo; e, finalmente, o terceiro objectivo é ver a adaptação que ponto tornou-se, se um total ou parcial. Para afirmar ou refutar estes obxectivos, a metodoloxía utilizada foi dobre, primeiro unha análise e revisión de documentos da literatura científica e outras análise cuantitativa - cualitativa a través dun experimento levado a cabo en recursos patrimoniais baixo cuestionario investigación.

Palavras - Chave: Catedral, Destino, Recurso, Acessibilidade Universal, Incapacidade

\section{INTRODUCCIÓN}

En estas dos primeras décadas del siglo XXI el llamado turismo cultural así como una de sus subcategoria , el turismo religioso se han consolidado como tipologías turísticas mostrándose además, en una constante evolución y crecimiento (Gil de Arriba, 2006). La necesidad de que los recursos que son objetivo de ser mostrables al público, es decir, que se convierten en recursos turísticos, sean accesibles es un planteamiento que algunos gestores culturales se están planteado; pero lo que no está tan claro es la viabilidad de que algunos de dichos recursos puedan ser adaptados a dichas necesidades (Bieler, 2008). Viajar por razones culturales nos permite conocer no sólo el arte, la historia, la literatura, la antropologia, etc, sino acercarnos un poco más a lo que somos como seres humanos, ayudándonos a fundamentar algunas prácticas e ideologías que adquieren un valor universal (Gil de Arriba, 2006) tal y como señala la propia definición que da la UNESCO de Patrimonio Mundial. Pero, si estamos hablando de un valor universal, ¿es universalmente accesible?

El turismo religioso se está mostrando como uno de los nichos del mercado turístico en constante evolución y crecimiento, según la OMT (2015) así, respecto al crecimiento a principios del siglo XXI el turismo religioso es el 20\% del turismo mundial (Lanquar, 2007), y respecto a la evolución, ésta se muestra tanto a nivel de infraestructuras, que se han ido adaptando a las necesidades y motivaciones de los turistas; al propio perfil del viajero, ya que

International Journal of Professional Business Review (JBReview), São Paulo V.2 N.1 2017, pp. 72-89, January/June 
si inicialmente eran turistas nacionales (personas mayores, grupos de jóvenes y familias) en la segunda década del siglo XXI evoluciona hacia un turismo más internacional, con estancias más cortas, demandando agencias especializadas, (OMT, 2015); como a los medios de comunicación e internet, de modo que cuando se busca por la red información sobre el turismo religioso, se pueden apreciar más de 4 millones de entradas en lengua castellana, 24 millones en inglés; 6,5 millones en italiano, etc.

De entre los edificios históricos, que se ubican en los centros históricos de los destinos maduros, se encuentran, tanto construcciones públicas como privadas: plazas, palacios, edificios monumentales, parroquias, iglesias, Catedrales, museos, entre otros. Evidentemente las motivaciones que llevan a los turistas a visitar dichos espacios son múltiples, pero en el caso de los edificios religiosos, la cuestión trataría de ver si realmente los flujos de turistas que acuden a dichos recursos patrimoniales-religiosos, son creyentes y practicantes de la fe que profesan y por tanto la visita a un edificio religioso es parte fundamental de su viaje, o bien si son sencillamente consumidores de un producto turístico (Cánoves-Valiente, 2006).

En el mundo existen más de 3 mil millones de creyentes, de los cuales aproximadamente 1,5 mil millones disponen de medios para hacer una peregrinación, así centros como Roma, la Meca, Israel, Santiago de Compostela, la India son destinos a los que acuden millones de turistas al año, (OMT, 2015). Pero delimitar claramente aquellos turistas que se mueven exclusivamente por motivos religiosos, los que lo hacen por motivos culturales y finalmente los que aúnan ambos, resulta complejo aunque si hay un elemento clave, el recurso o destino a visitar es muy especial y permite tomar la decisión e incluso la necesidad de acudir a él.

Los principales destinos, por tanto, los podríamos dividir en dos grandes grupos, aquellos que atraen a los plenamente devotos y que viajan sólo y exclusivamente movidos por la fe, y aquellos que por las peculiaridades históricas i/o culturales atraen a viajeros que se mueven por dichas peculiaridades o aquellos que aparte de conocer los espacios aprovechan para mostrar su respeto hacia unas creencias determinadas. De modo que, el turismo religioso ofrece a los turistas elementos tanto religiosos, culturales, paisajísticos, espirituales como tradicionales, y que no son espacios compactos sino que interactúan entre sí, lo que permite 
Turismo Religioso Y Accesibilidad Universal: Compatibilidades e Incompatibilidades

obtener esa variación de motivaciones anteriormente comentada. Ello implica que cada destino o recurso pasa a ser único, aunque puedan existir semejanzas, la especificidad las muestra a través de sus propias características que hacen que no se puedan ni alterar ni reproducir.

Viajar a lugares o recursos que configuran el turismo religioso, como por ejemplo: catedrales, monasterios, abadías, iglesias rurales o urbanas, capillas, templos, altares, entre otros, plantea la necesidad de regular cada vez más los flujos turísticos y dotar a dichos espacios de centros de acogida, servicios e infraestructuras destinadas a los visitantes, (Guimont, 1997). Pero no se trata sólo de un patrimonio tangible, es también un intangible, la fe, el amor a la arquitectura, el conocimiento, las tradiciones lo que finalmente configuran ese reclamo turístico (Abril, Azpelicueta, y Sánchez, 2014).

El proceso de "patrimonializar" para poder visitar un recurso implica un conjunto de acciones complejas que se pueden agrupar en tres grandes áreas: en primer lugar proteger y restaurar, en segundo la promoción y en tercero la adaptación al número de visitantes y es justo en esta tercera área donde las observancias para la accesibilidad no siempre son posibles. Es decir, se entiende que la persona que sufre alguna discapacidad tiene que tener reconocidos y garantizados los derechos, entre ellos el de igualdad de condiciones.

Pero ¿realmente los valores de igualdad, libertad e incluso podríamos añadir de solidaridad, entendidos como derechos fundamentales, se ven reflejados en la práctica turística cultural? Si bien la Ley 51/2003 de igualdad de oportunidades, no discriminación y accesibilidad universal de las personas recoge dichos principios los gestores de los recursos turísticos patrimoniales, ya sea por problemas económicos, ya sea por problemas inherentes al propio recurso se ven incapacitados para asumir totalmente lo que significan dichos valores. Dicha ley expone que todos los ciudadanos tienen derecho a una participación activa en sus comunidades y que las personas que sufren discapacidades no tienen por qué verla coartada y que es necesario que se entienda que son personas que "[...] tienen dificultades para satisfacer sus necesidades, que son normales, más que personas especiales con necesidades diferentes al resto [...] (Jefatura del Estado, 2003: 43188) 
Todas estas premisas nos llevaron a plantearnos un objetivo general y varios objetivos específicos. Respecto al objetivo general de este estudio es saber si la accesibilidad universal se da en los recursos patrimoniales históricos, y más concretamente en ciertas Catedrales del arco mediterráneo e inseridas en la etapa de la Corona de Aragón y Condado de Barcelona, durante el siglo XIV. Para ver dicho estado de la cuestión nos hemos marcado tres objetivos específicos: el primero dilucidar cómo gestionan las tres Catedrales objetivo de este estudio los problemas de motricidad del público en general, el segundo tener conocimiento sobre qué líneas de accesibilidad se ha hecho más hincapié: motriz, sordera o ceguera o si se han gestionado todas por un igual; y finalmente el tercer objetivo es ver en qué grado se ha hecho la adaptación, si de un modo total o parcial y por tanto si podemos hablar de accesibilidad universal en estos tres recursos.

En base a los objetivos planteados la metodología empleada se fundamenta en dos fases: La primera, un análisis y revisión documental de la literatura científica pertinente: libros, artículos de revistas y material propio que permita consolidar los conceptos de accesibilidad universal y que, además, permita argumentar a nivel teórico nuestros objetivos. La segunda, un análisis cuantitativo comparativo de los tres recursos. Para poder realizar un análisis sobre la accesibilidad en las Catedrales de Barcelona, Girona y Palma de Mallorca se partía de un estudio precedente en el cual se había planteado si las Catedrales de la Corona de Aragón y Condado de Barcelona podían llegar a ser un Recurso Turístico (Abril, Azpelicueta, \& Sánchez, 2014) la afirmación nos llevó a abrir nuevos estudios añadiendo no sólo nuevos recursos sino una nueva línea de investigación, la accesibilidad. Así pues, nacía un nuevo cuestionario dirigido directamente a conocer cómo se trataba el tema objeto de nuestra investigación en dichos recursos.

A través de un cuestionario de 25 preguntas se ha podido hacer un análisis comparativo de la gestión de los tres recursos para así poder determinar en qué grado evolutivo sobre la accesibilidad universal se encuentran dichos recursos, dilucidando qué elementos son o no comunes y hasta donde llegan las compatibilidades o incompatibilidades en su gestión. La elaboración y resolución de los cuestionarios se realizó entre diciembre de 2015 y marzo de 2016 y el Universo a tratar fueron tres Catedrales: la de Barcelona, la de Girona y la de Palma de Mallorca.

International Journal of Professional Business Review (JBReview), São Paulo V.2 N.1 2017, pp. 72-89, January/June 
Turismo Religioso Y Accesibilidad Universal: Compatibilidades e Incompatibilidades

Finalmente a través del análisis llegaremos a las conclusiones, que nos permitirán aseverar o refutar si en el caso de las tres Catedrales estudiadas se dan los principios y valores de accesibilidad universal. Además el estudio también nos permitirá conocer, en caso de que exista, qué tipo de adaptación se ha producido y sobre que tipologías de minusvalías se han producido.

\section{MARCO TEÓRICO: ACCESIBILIDAD UNIVERSAL Y RECURSO PATRIMONIAL}

En estas dos últimas décadas el término de accesibilidad ha ido evolucionando. Si inicialmente se abordaba como un término enclavado en el ámbito médico hoy en día representa un modelo social, donde sus cimientos son los derechos de las personas. En base a este concepto social, tanto el panorama normativo internacional como nacional se han ido haciendo eco de los cambios y de las nuevas interpretaciones.

Así en el ámbito internacional, la OMT aprobaba en el año 2005 una resolución que asentaba algunos de los criterios básicos de igualdad de oportunidades para las personas con discapacidades que era necesario tener en cuenta. Nueve años más tarde, en el 2014 la propia OMT indicaba que una de las metas más importantes que tenían que lograr los gestores turísticos era facilitar la accesibilidad, lo que se vendría a llamar facilitar un turismo accesible. Durante el itinere de la OMT, se dio la Convención de las Naciones Unidas del año 2007 que trataba sobre los derechos de las personas con discapacidad, mientras en el ámbito Nacional una de las Leyes a destacar por sus criterios y parámetros es la Ley 51/2003 de 3 de diciembre, donde se amparaban los derechos de igualdad de condiciones, de no discriminación y de accesibilidad universal. Así en su exposición de motivos la Ley 51/2003 señala

“[...] Las personas con discapacidad constituyen un sector de población heterogéneo, pero todas tienen en común que, en mayor o menor medida, precisan de garantías suplementarias para vivir con plenitud de derechos o para participar en igualdad de condiciones que el resto de ciudadanos en la vida económica, social y cultural del país [...]" (Jefatura del Estado, 2003: 43187)

International Journal of Professional Business Review (JBReview), São Paulo V.2 N.1 2017, pp. 72-89, January/June 
Pero esta Ley no se limita sólo a unos principios y un articulado sino que también se hace eco de la necesidad de definir qué se entiende por accesibilidad universal, y así en su artículo segundo apartado c) la define como:

“[...] La condición que deben cumplir los entornos, procesos, bienes, productos y servicios, así como los objetos o instrumentos, herramientas y dispositivos, para ser comprensibles, utilizables y practicables por todas las personas en condiciones de seguridad y comodidad y de la forma más autónoma y natural posible. Presupone la estrategia de «diseño para todos» y se entiende sin perjuicio de los ajustes razonables que deban adoptarse [...]"(Jefatura del Estado, 2003: 43187)

Por tanto, hablar de accesibilidad universal se refiere, en primer lugar, a la posibilidad que todas las personas puedan tener acceso, uso y disfrute de entornos, productos y servicios de manera normalizada, segura y eficiente, y en segundo lugar, a tratar de elaborar estrategias que permitan un diseño para todos y las medidas de apoyo que sean necesarias. En resumen, un concepto que engloba distintos ítems, puesto que la discapacidad va referida tanto a una deficiencia sensorial y/o física, (Campoy, 2004), psíquica (Dito, Prieto, y Yuste, 2004) y una general que responde a aquellas personas que en un momento determinado ven mermadas sus facultades, ya sea de manera temporal o permanente, como por ejemplo, ir con escayola, una operación ocular, llevar un carrito de bebe, o un andador, entre otras (De Lorenzo, 2003).

Para que las estrategias sean viables, tanto la sociedad civil en su conjunto como los poderes públicos y las empresas han de tomar medidas para las modificaciones que sean necesarias en el entorno, para que cualquier persona pueda hacer uso del mismo en esa igualdad de condiciones. Así, con el diseño se pueden llegar a construir espacios, productos y servicios para todos los ciudadanos independientemente de cuál sea su capacidad funcional, alcanzando la superación de las desventajas que pueden encontrarse las personas con discapacidad.

Pero a pesar de la ratificación hecha por parte de los países miembros de la Convención de las Naciones Unidas (2007), a menudo, los problemas de accesibilidad se siguen dando, desde distintos puntos de vista y en grados diferentes, en el propio recurso donde, de un modo $\mathrm{u}$ otro con mayor o menor fortuna, se intentan aplicar los principios anteriormente

International Journal of Professional Business Review (JBReview), São Paulo V.2 N.1 2017, pp. 72-89, January/June 
Turismo Religioso Y Accesibilidad Universal: Compatibilidades e Incompatibilidades

mencionados. Las personas con discapacidad pueden tenerla de manera parcial o total y por tanto, el origen de la misma se encuentra en sus dificultades personales, pero no es el único punto de origen, también se puede encontrar en los obstáculos y límites que la propia sociedad ha ido consolidando a lo largo de los años, en base a lo que se podría llamar "el patrón de la persona media".

La OMT emitía un informe en el año 2013 donde se reflejaba que un 15\% de la población mundial tenían algún tipo de discapacidad y advertía que ese porcentaje iría en aumento debido al aumento de las enfermedades crónicas, al envejecimiento de la población y a los accidentes de todo tipo (laborales, ocio, conducción, etc.). Con estas cifras vivir sin afrontar lo que puede significar a nivel turístico no tener adaptados los recursos e infraestructuras será muy probablemente un factor clave para adoptar una decisión u otra a la hora de escoger un determinado destino Puesto que uno de los nichos de mercado que va alcanzando un mayor protagonismo es el que hace referencia justamente al de las personas con discapacidades (Cánoves, Herrera, \& Villarino, 2014). Según el Presidente del Foro Europeo de Personas con Discapacidad Yanis Vardakastanis, la falta de accesibilidad no deja de ser una manera sutil de discriminación, y si bien las leyes pueden hablar de derechos y regularlos no es menos cierto que una parte de los productos, servicios y recursos aún les falta un largo recorrido por andar para alcanzar la no discriminación, es decir, la plena accesibilidad.

Las ofertas, por tanto que se presentarán podrán ser amplias, ya sea a nivel servicios, ya sea a nivel productos (De la Calle Vaquero, 1999) y por tanto ese consumidor de productos culturales deseará probar nuevas experiencias. Por su parte el turismo religioso se está convirtiendo en uno de los productos más populares en Europa y España (Nolan y Nolan, 1992) pero la pregunta que surge es ¿pueden los turistas acceder a dichos espacios por qué éstos están preparados para ello? Evidentemente el turismo se muestra como una actividad que, de un modo u otro, afecta, ya sea positiva o negativamente, tanto a los espacios como a los recursos donde se desarrolla, el gestionarlo de manera adecuada evita el proceso destructivo o modificativo que puede conllevar dicha actividad (Lo Fruscio, 2011). 


\section{ESTUDIO DE CASO: LAS CATEDRALES DE BARCELONA, GIRONA Y PALMA DE MALLORCA}

La Catedral como edificio religioso que es, se integra dentro del turismo religioso y obviamente en el turismo cultural, pero ¿es capaz de abrazar los conceptos de turismo accesible y responsable? No cabe duda que la Catedral es un recurso turístico vivo, y se transforma en un atractivo turístico más del destino en el que se haya (Abril-Sellares, M. y otros 2014), pero aun siendo importantes los conceptos de afirmación de recurso patrimonial, este estudio se focaliza en determinar su accesibilidad, bajo la nueva acepción de accesibilidad universal.

Los motivos por lo que se han escogido estos tres recursos y no otros son por un conjunto de factores comunes: se trata de construcciones medievales, de estilo gótico meridional, construidas durante el reinado de la Corona de Aragón y Condado de Barcelona, y se encuentran situadas en tres ciudades capitales provinciales y además en dos Comunidades Autónomas situadas en el ranquin de las más visitadas en España, Catalunya cerró el año 2015 con el 25,57\% del total, y las Illes Balears lo hicieron con el 17,1\% del total (Instituto Nacional de Estadística, 2016). Es importante señalar que este estudio tendrá continuidad en el tiempo dado que abarcará todas las Catedrales que se construyeron en el periodo histórico anteriormente mencionado.

Para poder realizar un análisis sobre la accesibilidad en las Catedrales de Barcelona, Girona y Palma de Mallorca se partía de un cuestionario de 25 preguntas que nos situaría en la realidad y estado de la cuestión.

En un primer testeo se pudo observar que algunos de los ítems que se incluían en la accesibilidad universal no se daban en ninguno de los casos a estudio, lo cual, nos confirmaba, de entrada, que en términos de gestión las tres Catedrales no cumplían algunos de los criterios de accesibilidad universal, ya fuera ésta referida a conceptos motrices, sensoriales o visuales, y que ninguna de las tres contemplaba los aspectos psíquicos. Ello hizo que nos centrásemos en las tipologías que podían mostrar el estado de la cuestión con datos

International Journal of Professional Business Review (JBReview), São Paulo V.2 N.1 2017, pp. 72-89, January/June 
Turismo Religioso Y Accesibilidad Universal: Compatibilidades e Incompatibilidades

cuantitativos, por tanto, se trabajó con la accesibilidad para las minusvalías motrices y sensoriales tanto auditivas como visuales.

Las preguntas del cuestionario se dividieron en tres grandes bloques, el primero, correspondiente a un grupo de 11 preguntas que hacía referencia a la accesibilidad motriz, tanto a nivel de entrada de los recursos como en el interior de los mismos. El segundo bloque abarcaba un total de 12 preguntas que se dirigía a conocer cómo gestionaban la accesibilidad sensorial tanto a nivel visual como auditiva. Este bloque a su vez se dividía en dos sub bloques, uno referido a gestión in situ y otra a través de la página web.

Los primeros ítems de la investigación iban referidos a los accesos que poseían las tres Catedrales y a conocer cuántos de ellos se habían adaptado. Los resultados obtenidos muestran, ya inicialmente, véase el Gráfico 1, la disparidad de criterios adoptados para la gestión. Ninguna de las tres Catedrales, tiene las entradas del recurso adaptadas al 100\% o lo que es lo mismo no todas las entradas tienen rampas que permiten el acceso al interior. Así la de Palma de Mallorca presenta una adaptación del 33\% mientras que la de Barcelona adapta el $60 \%$ de las mismas y Girona, el 66\% del total, cifras que quedan muy alejadas del concepto de accesibilidad universal recogido en la Ley 51/2003 de 2 de diciembre.

Tabla 1: Adaptación de las entradas de las Catedrales

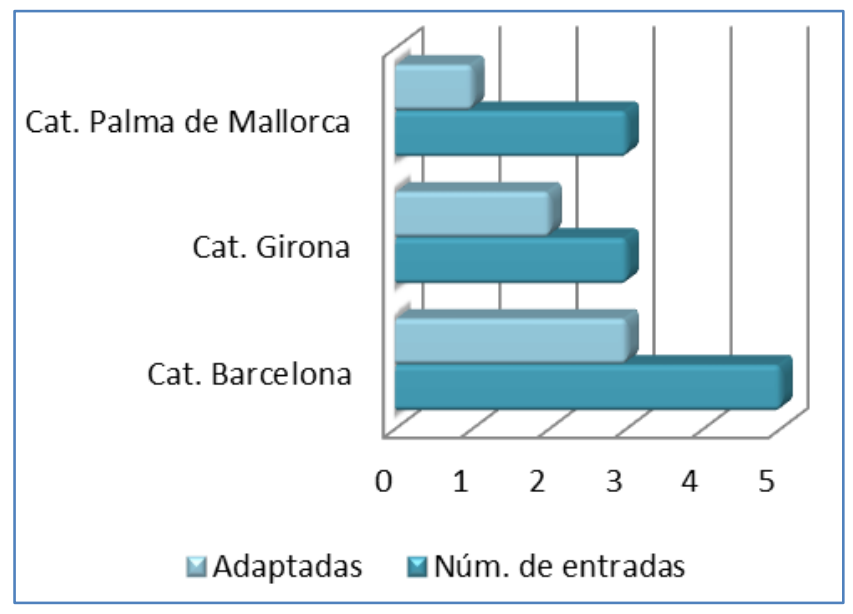

Fuente: Elaboración propia en base a los datos obtenidos del cuestionario

Dado que las tres Catedrales disponen interiormente de dos grandes espacios, el interior de la iglesia y el interior del claustro los siguientes ítems distinguían dichos espacios. Los 
resultados han mostrado que las tres Catedrales sólo adaptan una parte del espacio interior de la iglesia, allí donde aparecen suaves desniveles se opta por la opción de rampas en un 25\% en las tres Catedrales pero los espacios donde hay escaleras el 100\% no tienen ningún tipo de adaptación. Así tenemos que los espacios son las naves a las que sí se puede acceder, pero ni a la cripta, ni a las terrazas, ni a la galería y finalmente a las distintas capillas muestran una posibilidad de acceso, véase Tabla 2.

Por su parte los claustros siguen la misma tendencia que los interiores eclesiásticos, véase Tabla 2 ya que las zonas donde hay escaleras, es decir las capillas y las galerías en caso de existir, no son accesibles porque no están adaptadas, sólo la zona de las pandas permite una circulación relativamente fácil, aunque con unos pavimentos poco uniformes por las tumbas o relieves que en ellos se encuentran representados.

Tabla 2: Accesibilidad en el interior de las iglesias y en sus respectivos claustros

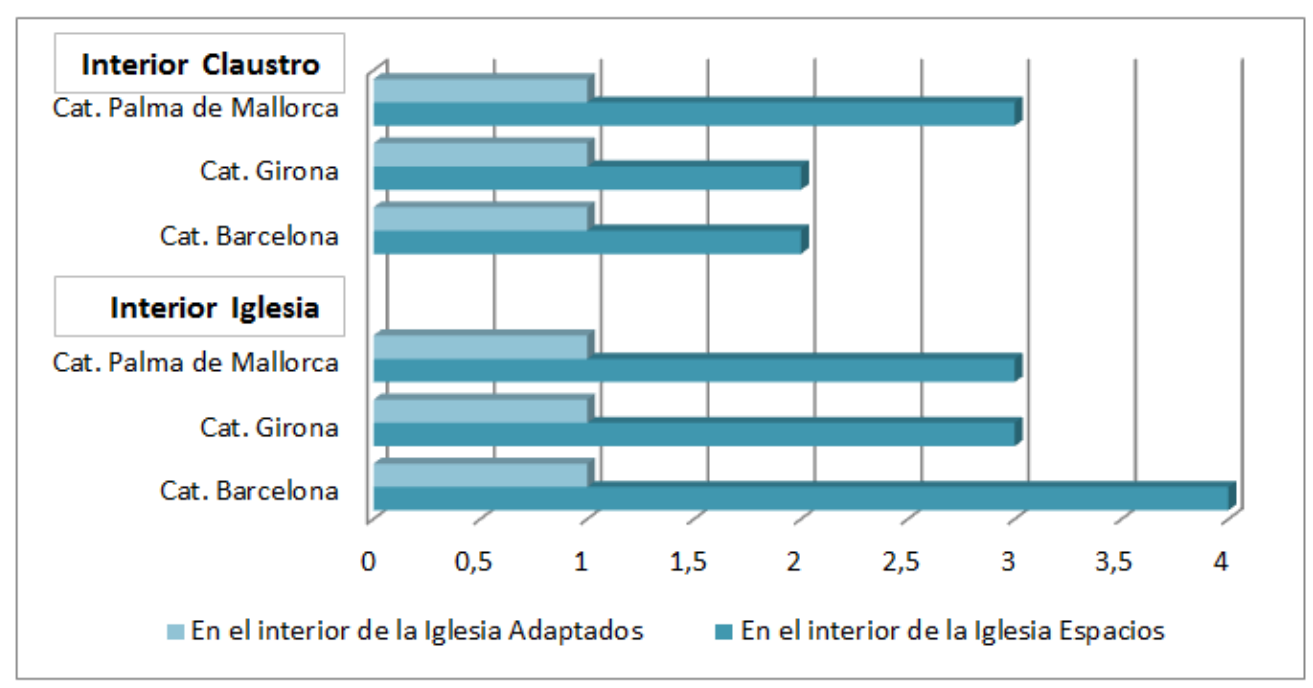

Fuente: Elaboración propia en base a los datos obtenidos del cuestionario

A parte de los servicios que se desprendían de las estructuras arquitectónicas era necesario observar otros servicios que podían ofrecer estos tres recursos catedralicios a nivel turístico, de modo que se hicieron preguntas muy concretas que dieron como resultado los siguientes valores: 
Turismo Religioso Y Accesibilidad Universal: Compatibilidades e Incompatibilidades

1. En estos últimos años, la gestión turística de las Catedrales permite acceder a niveles superiores (galerías, triforios, terrazas) en el interior de la Iglesia. Por tanto era importante saber si hay ascensores y que adaptación se había hecho en ellos. Del trabajo de campo observamos que sólo la Catedral de Barcelona posee ascensor pero que no está adaptado, lo que significaba que hay zonas de la construcción religiosa donde es inviable que personas con necesidades especiales motrices (con sillas de ruedas, o personas que lleven carritos de bebé) puedan conocer dichas zonas por no estar adaptado el sistema mecánico que los conduciría a las mismas.

2. Siendo un recurso turístico creíamos necesario saber si tenían o no, un servicio de WC, evidentemente adaptado, la Catedral de Barcelona y la de Palma de Mallorca tienen dicho servicio adaptado, mientras que la Catedral de Girona, no.

3. Respecto a la altura de las cartelas informativas la gestión de la misma en las tres Catedrales era similar, sólo una parte de las mismas está adaptada. No era fácil determinar el porcentaje por el volumen de informaciones que cada Catedral da de los valores existentes en ellas. Pero con datos aproximados observamos que para la Catedral de Barcelona la adaptación era de un 60\%, mientras que las otras dos Catedrales no alcanzaban al $40 \%$.

Las cartelas permitían cerrar el bloque de la accesibilidad motriz, pero además nos servían de enlace para el siguiente gran bloque referido a las preguntas de accesibilidad sensorial. En este caso, en ninguna de las tres Catedrales se había considerado el traducir al lenguaje Braile ninguna de sus informaciones, ni las fijas existentes dentro del recurso ni los folletos turísticos que se dan en la visita del mismo, véase Tabla 3. A ello hay que añadir que tampoco se muestra una señalización lumínica sustitutoria de guiaje para las personas que padecen ceguera parcial.

Finalmente, en este apartado, poner atención en el hecho de que si bien hay algunos sistemas de audio en los tres recursos turísticos ninguno tiene un sistema de subtítulos que ayuda a las personas con deficiencia auditiva De modo que a nivel sensorial el camino hacia la adaptación ni tan siquiera se ha iniciado, véase Tabla 3.

Tabla 3: Adaptación sensorial - visual y auditiva

International Journal of Professional Business Review (JBReview), São Paulo V.2 N.1 2017, pp. 72-89, January/June 


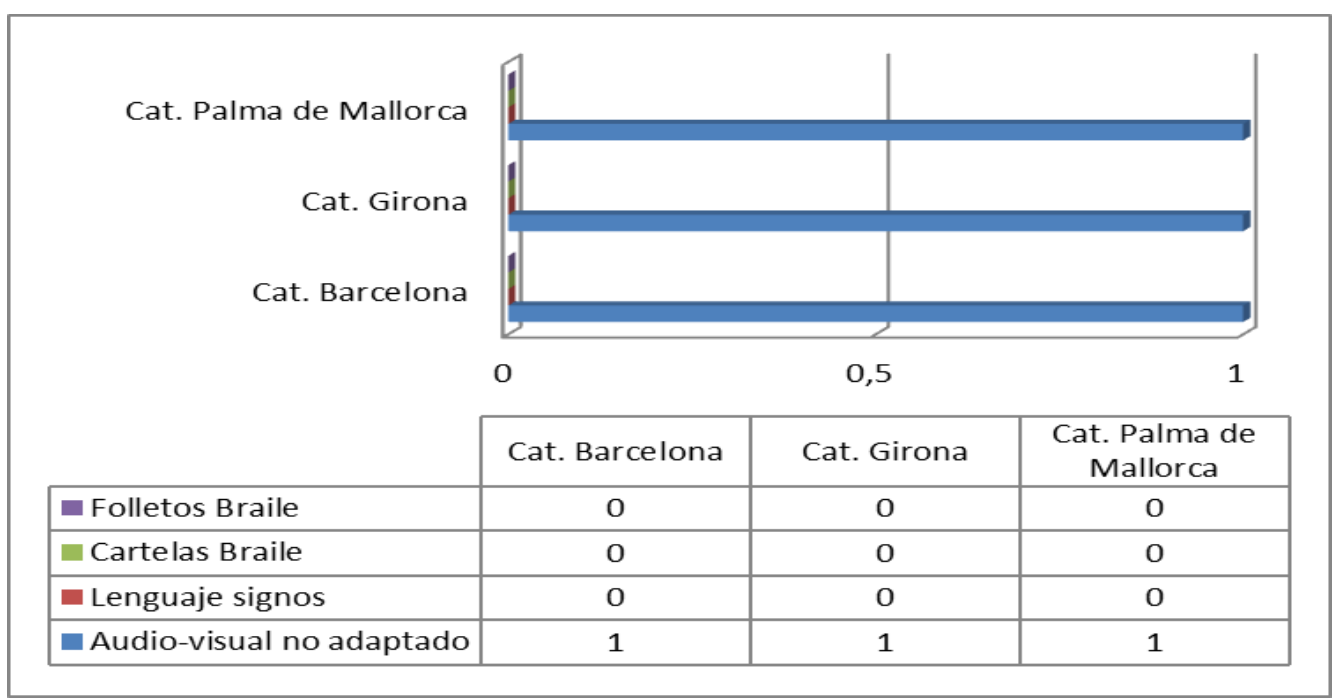

Fuente: Fuente: Elaboración propia en base a los datos obtenidos del cuestionario

La última parte del cuestionario se basaba en las páginas web de los dos recursos. De nuevo las tres Catedrales comulgaban en su gestión, en toda la página web no hay ningún tipo de adaptación, sólo se señala a través de iconos correspondientes si los recursos, una vez in situ, están o no adaptados, pero la página web, en sí misma considerada, no está pensada para personas con discapacidades sensoriales, concretamente de ceguera y en el caso de vídeos con personas que hablan o cantan con subtítulos para las personas que padecen sordera.

\section{CONCLUSIONES}

1. Después del análisis de los tres recursos, podemos afirmar que, a pesar que los tres recursos son considerados turísticos y que forman parte de las zonas históricas, los temas de accesibilidad están aún muy lejos de ser resueltos. Ninguno de los recursos tiene una adaptación del $100 \%$ ni respecto a la motricidad ni a la visualidad ni al sensorial. Estaríamos hablando que su adaptación no alcanza ni tan siquiera el 20\% en ninguna de ellas, ya que la falta absoluta de adaptación en temas sensoriales y visuales lleva a la baja el porcentaje de la motricidad dejando una adaptación cercana al 25\%, lo cual dista muy lejos de los valores que se determinan de forma legal.

2. Evidentemente se han hecho algunas actuaciones pero que hemos de calificar de totalmente insuficientes. Sólo la Catedral de Palma, tiene adaptados todos sus accesos externos a nivel motriz, el uso de rampas se puede considerar por tanto insuficiente pero no 
Turismo Religioso Y Accesibilidad Universal: Compatibilidades e Incompatibilidades

imposibilita para que personas con su capacidad motriz disminuida no puedan acceder al recinto. Internamente, el discurso cambia, y no precisamente a una concepción positiva, acceder a capillas, galerías, triforios, criptas, salas capitulares u otros espacios, se hace casi imposible. Las personas con motricidad reducida, se ven abocados a ver las Catedrales de manera sesgada, lo que puede conducir a una cierta insatisfacción e incluso frustración. Por tanto, podemos determinar que la gestión turística en cuanto a términos de accesibilidad motriz universal, por parte de las tres Catedrales está aún en "pañales" y que requerirá de un gran esfuerzo tanto técnico como económico en el caso de que se quiera alcanzar unos niveles mínimos que se puedan considerar adecuados. Hablar de menos de un 50\% es lo que nos lleva a afirmar que la gestión turística no es, hoy por hoy, la adecuada.

3. En cuanto a la accesibilidad para minusvalías sensoriales, la gestión llevada a cabo es totalmente inexistente. En ninguna de las tres Catedrales se ha podido apreciar que las cartelas tengan una altura adecuada para la lectura y que presenten el lenguaje Braile para poder entender lo que dicen. Tampoco hay zonas lumínicas o bandas rugosas para personas con ceguera parcial, y en el caso de los audios (Barcelona) no están ni subtitulados ni utilizan el lenguaje de los signos. De modo, que podemos afirmar que para los segmentos poblacionales que padecen alguna disminución física parcial o total, temporal o permanente es totalmente impensable conocer el recurso turístico tal y como pueden conocerlo otras personas que no padecen de dichas minusvalías.

De momento, las tres Catedrales, han abocado sus esfuerzos a una de las minusvalías, la motriz, así que quizá abrir su campo de miras hacia las otras minusvalías sería necesario para dar no sólo mayor confianza sino acercarse a lo que teóricamente las normas tanto constitucional como leyes defienden.

4. Por otro lado las páginas web en sí mismas no están pensadas o dirigidas a personas con minusvalías. Lo único que aparece como información es si el recurso se encuentra adaptado a las personas con movilidad reducida. Es imprescindible entender que una información adecuada en las páginas web permitiría tener, a una persona que sufre algún tipo de discapacidad, todos los criterios para saber si el recurso es accesible o no en su caso 
particular, lo cual determinaría la compatibilidad del recurso con los problemas de accesibilidad universal.

Si la información es la adecuada y se adapta a las necesidades muy probablemente el público, que padece de modo total o parcial, permanente o temporal alguna minusvalía, se sentirá más atraído a visitar los espacios donde no sólo su acceso sino su información llegan a este segmento poblacional sin problemas.

Finalmente concluir en que si bien se ha trabajado por parte de los gestores turísticos de los tres recursos el problema de la accesibilidad, bajo un concepto casi exclusivamente de rampas y de algún servicio como pueden ser los baños públicos, es evidente que no es suficiente, y que sólo su adaptación se da en términos de parcial y sesgado.

\section{REFERENCIAS BIBLIOGRÁFICAS}

Abril, M., Azpelicueta, C., \& Sánchez, M. (2014). “Turismo religioso y destinos maduros. La gestión turística de dos catedrales: Palma de Mallorca y Barcelona”. VII Jornadas de investigación en turismo, cultura, desarrollo y nuevas tecnologías (págs. 211218). Sevilla: Red de Impresión 2014 S.L.

Bieler. (2008). Desafío para el desarrollo social e inclusivo. Primer Congreso Interamericano y Tercer seminario nacional sobre discapacidad y derechos humanos. Panamá.

Campoy, I. (2004). "Una aproximación a las nuevas líneas de Fundamentación de los derechos de las personas con Discapacidad". Revista Telemática de Filosofia del Derecho, 125-155.

Cánoves, G., Herrera, L., \& Villarino, M. (2014). Integración, turismo y discapacidad: ¿son accesibles los hoteles para las personas con discapacidad física? Pasos. Revista de turismo y patrimonio cultural, 12(1), 2009-218.

Cánoves-Valiente, G. (2006). Turismo religioso en Montserrat: Montaña de Fe, Montaña de Turismo. Cuadernos de Turismo, 63-76. 
De Lorenzo, R. (2003). "El futuro de las personas con discapacidad en el mundo". ONCE. Madrid: Umbral.

Dito, E., Prieto, E., \& y Yuste, J. (2004). "El ocio y tiempo libre como claves integradoras en el deficiente mental". Revista interuniversitaria de formación del profesorado(18), 181-194.

Gil de Arriba, C. (2006). Turismo de Peregrinación y Turismo religioso: el valor sagrado de los lugares. Cuadernos de Turismo, 77-102.

Guimont, D. (1997). Quand les lieux de pèlegrinage s'ouvrent au tourisme...: les hauts lieux de pèlegrinage, la culture et le tourisme. Téoros, 16(2), 30-33.

IDOM, Ingeniería de Sistemas. (2011). El turismo cultural en el Mediterráneo: algunas oportunidades para España, Francia, Marruecos y Túnez. Málaga: Cámara de Comercio de Málaga.

Instituto Nacional de Estadística. (2016). Estadística de Movimientos Turísticos en Fronteras.2015. Madrid: INE.

Jefatura del Estado. (3 de diciembre de 2003). Ley 51/2003, de 2 de diciembre, de igualdad de oportunidades, no discriminación y accesibilidad universal de las personas con discapacidad. BOE. Madrid, Madrid, España: BOE.

Juncà, J. (2011). Accesibilidad universal al patrimonio cultural. Fundamentos, criterios y pautas. Madrid: Centro Español de Documentación sobre Discapacidad.

Lanquar, R. (2007). Conferencia Internacional de Córdoba sobre "Turismo y REligiones: una contribución al dialogo de religiones, culturals y civilizaciones". La nueva dinámica del turismo religioso y espiritual. Córdoba: OMT.

Lo Fruscio, M. (2011). Estudio de turismo accesible: para discapacitados motrices en la ciudad de Cuenca" (Tesis Doctoral). Cuenca, Ecuador.

Naciones Unidas. (2007). Convención sobre los derechos de las personas con discapacidad. Nueva York: Naciones Unidas. 
Nolan, M., \& Nolan, S. (1992). Religious sites as Torism Attractions in Europe. Annals of Turourism Research, 68-78.

OMT. (2005). Hacia un turismo accesible para todos. Madrid: UWNTO.

OMT. (2013). Accesibilidad: una ventaja competitiva en los destinos turísticos. Madrid: OMT.

OMT. (2014). Mnual de turismo accesible para todos: Alianzas público-privadas y buenas prácticas. Madrid: UWNTO.

OMT. (2015). Turismo y religiones. Una contribución al diálogo entre religiones, culturas y civilizaciones. Córdoba: UNWTO.

Parellada, J. (2009). Conferencia Episcopal Española. El Turismo religioso. Sus perfiles (págs. 2-27). Avila: OSB.

PREDIF. (2014). Guía de Buenas Prácticas de Accesibilidad para los Recursos Turísticos de las Ciudades Patrimonio. Ávila: Ministerio de Educación, cultura y deporte.

SEGITUR. (2014). Spain info. Recuperado el 28 de agosto de 2014, de Turismo Accesible: http://www.spain.info/es/informacion-practica/turismo-accesible/

Westcott, J. (2005). Mejorar la información sobre el turismo accesible para las personas con discapacidad. Luxemburgo: Oficina de Publicaciones Oficiales de las Comunidades Europeas.

\section{INDICE DE TABLAS}

Tabla 1: Adaptación de las entradas de las Catedrales................................................82

Tabla 2: Accesibilidad en el interior de las iglesias y en sus respectivos claustros........83

Tabla 3: Adaptación sensorial - visual y auditiva ................................................ 84

International Journal of Professional Business Review (JBReview), São Paulo V.2 N.1 2017, pp. 72-89, January/June 